\title{
Acceleration and isobaric separation of radioactive ion beams with the Louvain-la-Neuve isochronous cyclotrons
}

\author{
M. Loiselet, N. Postiau, G. Ryckewaert \\ Centre de Recherches du Cyclotron \\ Université Catholique de Louvain \\ 2 Chemin du Cyclotron, B-1348 Louvain-la-Neuve, Belgium \\ A. Morduev, R. Oganessian \\ Flerov Laboratory, JINR, Dubna, Russia
}

\section{Abstract}

The paper reports on the results obtained for the production and acceleration of radioactive ions with the Louvain-la-Neuve cyclotrons. The specific problems of the separation of isobaric beams at low energy with a high efficiency is presented. Their consequences on the design of a cyclotron dedicated to the acceleration of radioactive beams are discussed.

\section{INTRODUCTION}

The Radioactive Ion Beam facility at Louvain-laNeuve aims at the acceleration of unstable nuclei $\left(\mathrm{T}_{1 / 2} \leq 2 \mathrm{~h}\right)$ in the low energy range $(<2.0 \mathrm{MeV}$ per nucleon), which is of interest for cross section measurements of important reactions involved in astrophysical processes. It uses two cyclotrons coupled by an on-line ECR source to produce, ionize and accelerate the radioactive species. With this scheme, intensities of $10^{6}$ particles per second (pps) for ${ }^{6} \mathrm{He}$ and up to $10^{9} \mathrm{pps}$ for ${ }^{19} \mathrm{Ne}$ have been obtained. Although the acceleration efficiency is low at these energies $(<5 \%)$, it turns out that the high mass resolving power of a cyclotron is crucial to eliminate the isobaric contamination in a very efficient way. Results obtained so far will be presented and the main design feature of a new cyclotron dedicated to the acceleration and isobaric separation of radioactive ions will be discussed.

\section{ACCELERATION OF RADIOACTIVE IONS IN CYCLONE}

\subsection{General layout}

The general layout of the facility has been presented elsewhere [1]. The high intensity $(200 \mu \mathrm{A}) 30 \mathrm{MeV}$ proton beam at a first cyclotron, CYCLONE 30, is used to produce large amounts of radioactive atoms in a target located in the wall which separates the two vaults. These are pumped out of the target through an Electron Cyclotron Resonance (ECR) ion source in which they are ionized. After a first analysis in a low resolution mass separator, ions with the same mass to charge ratio are injected in the second cyclotron, CYCLONE, which brings them to the required energy $[1,2]$.

\subsection{Acceleration and isobaric separation}

Most of the elements that have been accelerated so far, have an isobaric contaminant coming either from the target or from the residual gas in the source. To achieve a high purity in the final beam, the cyclotron is tuned as a high resolution spectrometer and the intensity of the isobaric beam is considerably reduced after the acceleration and extraction processes. For isobars with a relative mass difference of $2 \times 10^{-4}$, like $\left({ }^{19} \mathrm{Ne},{ }^{19} \mathrm{~F}\right)$ or $\left({ }^{11} \mathrm{C},{ }^{11} \mathrm{~B}\right)$, an attenuation factor of the order of $10^{6}$ is routinely achieved with only a minor reduction in the acceleration efficiency. More details about this were given elsewhere [3].

Table 1 summarizes the intensities and the maximum energy available for the various ions that have been delivered by CYCLONE. Most of them have been accelerated at energies between 0.6 and $2 \mathrm{MeV} / \mathrm{Nucleon}$ (which is the energy domain of interest for nuclear astrophysics) and intensities larger than $10^{9}$ pps were obtained for ${ }^{19} \mathrm{Ne}^{2+}$. However, as soon as high charge states are provided by the ECR source, higher energies can be reached as it has been shown with ${ }^{19} \mathrm{Ne}^{4+}$ which was accelerated at $4.2 \mathrm{MeV} / \mathrm{amu}$ with an intensity of $5 \times 10^{8}$ pps.

Table 1

Intensities and energies of the radioactive beams which are presently available at the Louvain-la-Neuve facility

\begin{tabular}{lllcc} 
Element & \multicolumn{1}{c}{$\mathrm{T}_{1 / 2}$} & $\mathrm{q}$ & $\begin{array}{c}\text { Intensity } \\
(\mathrm{pps})\end{array}$ & $\begin{array}{c}\text { Max. Energy } \\
(\mathrm{MeV})\end{array}$ \\
${ }^{6} \mathrm{He}$ & $0.8 \mathrm{~s}$ & $1^{+}$ & $1.210^{6}$ & 18 \\
$11_{\mathrm{C}}$ & $20 \mathrm{~min}$ & $1^{+}$ & $1.010^{7}$ & 10 \\
$13_{\mathrm{N}}$ & $10 \mathrm{~min}$ & $1^{+}$ & $4.010^{8}$ & 8.5 \\
& & $2^{+}$ & $3.010^{8}$ & 34 \\
& & & \\
$18 \mathrm{Ne}$ & $1.7 \mathrm{~s}$ & $3^{+}$ & $4.210^{5}$ & 55 \\
${ }^{19} \mathrm{Ne}$ & $17 \mathrm{~s}$ & $2^{+}$ & $1.910^{9}$ & 23 \\
& & $4^{+}$ & $5.010^{8}$ & 93 \\
${ }^{35} \mathrm{Ar}$ & $1.8 \mathrm{~s}$ & $5^{+}$ & $\sim 10^{5}$ & 79
\end{tabular}




\section{DESIGN FEATURES OF A CYCLOTRON DEDICATED TO THE RADIOACTIVE BEAMS}

The reasons which led to the decision to build a new cyclotron (provisionally called CYCLONE44) for the acceleration of radioactive isotopes of the light elements for the study of nuclear reactions of astrophysical interest have already been discussed elsewhere $[2,3]$ and can be summarized as follows:

- its energy range: from 0.2 to $0.8 \mathrm{MeV} / \mathrm{AMU}$, covering the astrophysically important region not accessible with CYCLONE (the lower limit is $0.56 \mathrm{MeV} / \mathrm{AMU}$ );

- its acceleration efficiency: one order of magnitude larger than the efficiency of CYCLONE allowing the study of nuclear reactions with smaller cross sections, e.g. those reactions involving alpha particles and short lived radioactive nuclei;

- its isobaric resolving power: $10^{4}$ to allow the use of pure radioactive ion beams of very low intensity in the presence of large isobaric stable element beams;

- its availability and flexibility: for the production of radioactive nuclei, both CYCLONE30 (as in the actual case) and CYCLONE, with its greater variety of particles and higher energy but lower intensity will become available. The new dedicated cyclotron will be full time available for long term measurements.

The main characteristics of CYCLONE44, resulting from a detailed study of the experiments to be performed with it have been fixed now and are given in table 2 .

Table2

Main characteristics of CYCLONE44.

$\begin{array}{ll}\text { Energy constant K (MeV) } & 44 \\ \text { Energy range (MeV/AMU) } & 0.2-0.8 \\ \text { Mass range } & 4-30 \\ \text { Maximum avcragc ficld (T) } & 1.5 \\ \text { Extraction radius (m) } & 0.64 \\ \text { RF - system } & 2 \text { Dees, var. angle } \\ \text { Frequency range (MHz) } & 12-17.5 \\ \text { Maximum Dee voltage }(\mathrm{kV}) & 20 \\ \text { Harmonic modes } & 6-8 \\ \text { Injection } & \text { Axial } \\ \text { Extraction } & \text { Electrostatic }\end{array}$

\section{Description}

The magnet has four sectors and a cylindrical return yoke, similar to CYCLONE30: such a design leads to a reduced size and a reduction in weight of about $25 \%$ for a given $\mathrm{K}$-value, compared to the classical magnet. To further reduce the size and the power consumption, rather small hill and valley gaps have been choosen. Trimcoils will be located under the sectors and the accelerating electrodes will be located in opposite valleys. The most difficult area in obtaining isochronous fields is in the centre: the size of the axial hole is no longer small compared to the average gap and a smooth transition between the cylindrical centre plug and the sectors has to be realized at the earliest possible radius to assure vertical focusing. The magnet parameters are given in table 3.

Table 3

Magnet parameters of CYCLONE44

\begin{tabular}{|c|c|}
\hline Weight ( $\mathrm{T}$ & \\
\hline Height (m) & \\
\hline Outer diam & \\
\hline Pole diame & \\
\hline Hill gap (c & \\
\hline Valley gap & \\
\hline Sector ang & grees) \\
\hline Maximum & ield (T) \\
\hline Maximum & $y$ field $(T)$ \\
\hline Main coil: & $\begin{array}{l}\text { number of turns } \\
\text { maximum current }(\mathrm{A})\end{array}$ \\
\hline & maximum voltage $(\mathrm{V})$ \\
\hline
\end{tabular}

Correction coils : circular, under sectors

The main challenge in the design of CYCLONE44 lies in the combination of two requirements: a high acceleration efficiency (from the ECR source for multiply charged ions with a relatively large emittance, through injection, acceleration and extraction) and a high resolving power for isobaric contaminant beams.

The resolving power of a cyclotron is proportional to the number of turns times the acceleration harmonic mode number [3], thus asking for low Dee voltage and high harmonic modes. On the other hand, a large acceleration efficiency can only be obtained if the axially injected low energy beam is perfectly matched to the cyclotron centre region acceptance, in six dimensional phase space. This requirement calls in turn for low harmonic modes, high injection voltage and high Dee voltage.

These conflicting requirements were partially circumvented in CYCLONE by adjusting the radial field profile to be non - isochronous resulting in an increased turn number. This method is not too satisfactory because difficult to control and leading to sensitive and unstable beams.

For CYCLONE44, harmonic modes 6 and 8 have been choosen and the option was taken to vary the Dee angle radially. Along the first turn, the Dee angle is close to the optimum for maximum encrgy gain and posts are used to reduce the transit times in the gaps. Then the Dee angle is gradually reduced with increasing radius. This way, the effective energy gain per turn is kept small to assure the required turn number (resp. 265 in $\mathrm{H}=6$ and 200 in $\mathrm{H}=8$ ). To provide maximum turn separation at extraction the Dee angle is allowed to increase to optimum during the last $10 \mathrm{~cm}$ before extraction radius. The exact profile of the acceleration electrode and gap has to be optimized carefully to preserve longitudinal beam quality: decreasing energy gain with radius increases bunch length, increasing energy gain shortens the bunch but both phenoma do not cancel totally [4].

Particles are injected with a magnetic bending radius of $35 \mathrm{~mm}$ in the isochronous field and deflected in the median plane on a centered orbit with a spiral inflector. 
Extraction is performed using an electrostatic deflector eventually followed by a passive focusing channel if required. The turn separation at extraction is increased by an adjustable first harmonic field bump.

Good vacuum ( $10^{-7}$ range) is mandatory to minimize beam losses. The sectors and correction coils are in a primary vacuum to allow for light top and bottom covers of the vacuum chamber. The high vacuum in the acceleration chamber is obtained with diffusion pumps connected at the rear end of the Dee stem and with cryopumps connected through the return yoke.

The RF-system consists of two Dees connected together at the centre and coaxial resonators mounted vertically.

Figure1 shows a schematic section through the midplane of the cyclotron.

\section{REFERENCES}

[1] G. Berger et al., "Acceleration and mass-separation of radioactive ion beams in an isochronous cyclotron", 1991 IEEE Particle Accelerator Conference, San Francisco, pp 2610-2612.

[2] P. Decrock et al., '"Production and acceleration of radioactive ion beams. Proceedings of the Second International Conference on Radioactive Beams, Louvain-la-Neuve, 1991, edited by Th. Delbar (Adarn Hilger) pp 121-126.

[3] G. Ryckewaert et al., "Radioactive ion beam production using the Louvain-la-Neuve cyclotrons", Proceedings of the 13th International Conference on Cyclotrons and their Applications, Vancouver, 1992, edited by G. Dutto and M.K. Craddock.

[4] A. Chabert, private communication.

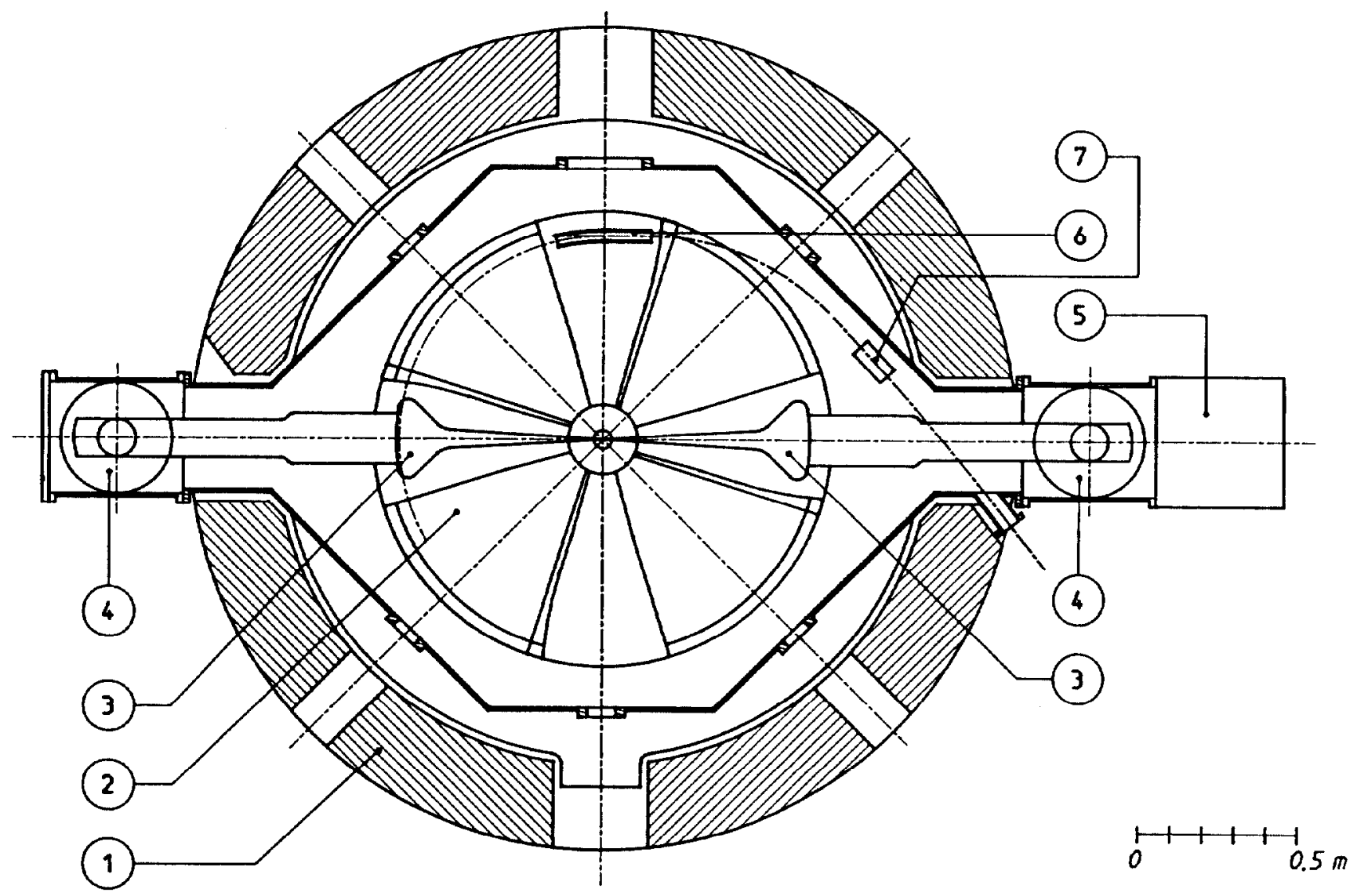

Figure 1

Schematic view of the midplane section of CYCLONE44

1. Magnet yoke 2. Sector 3. Accelerating electrode 4. Coaxial resonator 5. RF-amplifier 6. Electrostatic deflector 7. Passive magnetic channel - focusing 